\title{
Evaluation of the bacterial frequency causing ventilator associated pneumonia(VAP) in patients admitted in internal ICUs and internal wards at Shiraz Teaching Hospitals
}

\section{Tahereh Khalili}

Shiraz University of Medical Sciences

Nasser Samadi

Shiraz University of Medical Sciences

Milad Shahini Shams Abadi

Shahrekord University of Medical Science

Mohammad Ali Davar Panah

Shiraz University of Medical Sciences

Mohammad Motamedifar

Shiraz University of Medical Sciences

Nahal Hadi ( $\square$ nahalhadi.shz@gmail.com )

Research note

Keywords: Ventilator associated pneumonia, Hospital infections, Antibiotic susceptibility

Posted Date: August 21st, 2019

DOI: https://doi.org/10.21203/rs.2.13341/v1

License: (c) (i) This work is licensed under a Creative Commons Attribution 4.0 International License. Read Full License 


\section{Abstract}

Objective Ventilator associated pneumonia (VAP), which is a subset of hospital infections, occurs in patients who have been mechanically ventilated for at least 48 hours. The aim of this study was to determine the frequency of bacteria causing VAP and determine their antimicrobial susceptibility pattern.Results The present study is a cross-sectional descriptive study which was conducted in Shiraz during the 8 months (from November 2017 till June 2018). Samples were identified according to the diagnostic tests of bacteriology and antibiotic susceptibility test was performed using CLSI standards. 51 patients with VAP were examined, of which 10 and 8 patient respectively had chronic obstructive pulmonary disease and acute respiratory distress syndrome. The late onset VAP (61.7\%) rate was more than compared to the early onset VAP rate (38.3\%). The most frequently used antibiotics were meropenem (76.6\%) and vancomycin (78.7\%). Among 59 bacteria were isolated, Acinetobacter and Pseudomonas were the most prevalent organisms. Our data showed that most of the isolates (40\%) belonged to Multi Drug Resistant (MDR) pathogens.

\section{Background}

Ventilator associated pneumonia (VAP), which is a subset of hospital infections, occurs in patients who have been mechanically ventilated for at least 48 hours [1, 2]. VAP is considered a common, serious, costly complication in hospitalized patients, which in the intensive care units occupies the first rank of hospital infections and has a high mortality rate [3]. That's occurs between 48 to 72 hours after the tracheal intubation, is introduced as early onset pneumonia; this type of pneumonia is often a result of aspiration, which complicates the process of intubation and is often formed due to the bacteria sensitive to antibiotics (for example, Methicillin-Sensitive Staphylococcus Aureus (MSSA), Homophiles influenza and Streptococcus pneumoniae) [4].

Risk factors related to hospital acquired pneumonia include: age of over 45 years, heart ischemic disease, chronic obstructive pulmonary disease, kidney failure, dialysis, addicts to narcotic drugs, receiving antibiotic before the occurrence of pneumonia, smoking, previous hospitalization, coma, diabetes, blow to head, nasogastric tube, corticosteroid consumption, tracheostomy, mental status disorder and mechanical ventilation $[4,5]$. To diagnose etiology, a positive culture of lower respiratory tract secretions is needed. The most important sampling techniques are endotracheal tube aspiration (ETTA) and Bronchoalveolar lavage (BAL) and Protected Specimen Brushing (PSB). Long-term treatment can lead to colonization with resistant to antibiotics bacteria. Patients who have received a shorter period of antibiotic treatment showed better clinical outcomes than those with longer-period treatment [6]. Regarding the fact that day by day the MDR strains are increasing, and its high mortality rate of VAP, awareness of the occurrence of ventilator-associated pneumonia, identification of the causing pothogens and their antimicrobial susceptibility tests are necessary to improve and promote the hospital infection control system, and to employ effective methods of prevention, control, improvement of empirical treatments, reducing VAP incidence and the mortality resulting from it seems necessary. 


\section{Methods}

\section{Specimen collection, Identification of bacteria and Antimicrobial susceptibility test}

The present study is a descriptive cross-sectional study which was performed on all patients who had been hospitalized in the internal wards and ICUs of educational hospitals of Shiraz they had taken VAP during their hospitalization at the period of 8 months (from November 2017 to June 2018). The aim of this study was to determine the frequency of bacteria causing VAP and determine their antimicrobial susceptibility pattern.

All patients who had no symptoms of pneumonia at the time of intubation and who had pneumonia at least 48 hours after intubation, were included in the study. The diagnostic criteria for having VAP were considered in presence of at least two of the following cases: Based on fever above $38^{\circ}$ Celsius (hypothermia), leukocytosis or leukopenia, the presence of respiratory purulent secretions, the presence of new filtration or progressive in the chest radiographic [3].

Patient's endotracheal tube aspiration inside the crochet Falcon under sterile conditions were transferred to the laboratory of medical school. After transferring to the laboratory, the specimens were cultured and isolated on Blood Agar, MacConkey Agar (in aerobic atmosphere) and Chocolate Agar (with 5-10\% CO2 pressure). To detect Gram-positive cocci according to the gram positive cocci chart: from catalase test, coagulase test, Mannitol Salt Agar medium, DNase medium, Oxidase Test, differential. Antibiotic susceptibility tests by using Bacitracin, Optochin and Novobiocin Disks, Bile esculin test, $(6.5 \% \mathrm{Nacl})$ and hemolysis type $(a, \beta, \gamma)$ were used.

API $20 \mathrm{E}$ kit was used to detect gram negative bacilli, but, in order to detect gram-negative nonfermentative bacteria such as Acinetobacter and Pseudomonas species, differential culture media were separately prepared and tested for samples. In addition, the TSI medium were used for all gram-negative bacteria to examine the lactose and glucose fermentation pattern, gas and H2S production, and the SIM medium for motility of bacteria.

For final detection of the negative oxidase and fermentative sugars gram negative bacilli (Enterobacteriaceae) from the website of APIWEB: WWW.biomerieux-usa.com/clinical/api and nonfermentative gram-negative bacteria (such as Pseudomonas and Acinetobacter) from the website of ABSonline: WWW.tgw1916.net were used.

\section{Molecular detection}

To verify the species of Acinetobacter Baumannii, a molecular confirmation of PCR test on the bla OXA21 gene with primers F: 5'-TAATGCTTTGATCGGCCTTG-3' and R: 5'-TGGATTGCACTTCATCTTGG-3' was used [7].

Disk diffusion (Kirby Bauer) method was used to test the sensitivity of the samples to different antibiotics.. Antibiotics that were used for antibiogram listed in Table 1. The results of inhibition zone 
were interperate according to CLSI 2018 standards and SPSS16 software used for Statistical Analysis.

\section{Results}

\section{Basic information}

51 patients with VAP (hospitalized in the internal wards and internal ICUs of Shiraz educational hospitals) were sampled. after culturing, 47 bacteria and two yeast growed in media, and two samples did not grow. The average age of patients was 66 (range, 25-98 years), the average hospitalization period was 13.44 days (range, 3-45 days), and the average mechanical ventilation period was 11.73 days (range, 2-45 days). In this study, late onset VAP (61.7\%) was more prevalent than early onset VAP (38.3\%). The average calculated CPIS for patients was 8 (range, 6-10). The most commonly used empirical antibiotics were meropenem $(76.6 \%)$ and vancomycin $(78.7 \%)$. These two antibiotics were used in most cases as combined antibiotics. Information on the primary antibiotic treatment are presented in Table 2.

\section{Results for organisms causing VAP and antimicrobial susceptibility test}

Overall 59 bacteria that were isolated from 51 patients, 44 were gram negative and 15 were gram positive. $14(30 \%)$ of them were poly-microbial and $33(70 \%)$ were single - microbial. The number of these bacteria are presented in diagram 1. According to this chart, the most prevalent organisms were Acinetobacter Baumannii and Pseudomonas aeruginosa. In this study, also 4 methicillin-resistant Staphylococcus aureus were isolated. For diagnosis of MRSA, $30 \mu \mathrm{g}$ cefoxtin disk were used. Nineteen of the isolated bacteria were related to the Acinetobacter species, 17 of which belonged to Acinetobacter baumannii, and were confirmed by molecular PCR method and using bla oxa-51 like replication gene (Fig. 1). The results of the antimicrobial susceptibility of the Enterobacteriaceae family (14 bacteria) showed, the highest sensitivity of Enterobacteriaceae family belonged to meropenem (11 bacteria), imipenem ( 8 bacteria) and ciprofloxacin ( 8 bacteria). The highest antimicrobial susceptibility of Pseudomonas species(11 bacteria) was to meropenem ( 8 bacteria), imipenem ( 7 bacteria), amikacin ( 6 bacteria) and ciprofloxacin ( 6 bacteria) And the highest resistance to piperacillin tazobactam (7 bacteria), ceftazidime (9 bacteria) and cefepime ( 6 bacteria) and the highest Antimicrobial susceptibility of Acinetobacter species (19 bacteria) was observed in relation to meropenem (11 bacteria) and amikacin (11 bacteria) and the highest resistance in relation to SXT (13 bacteria), minosylcine (13 bacteria) and ceftazidime (13 bacteria). The antimicrobial susceptibility test of the gram-positives shown that the highest sensitivity was to Linezolid and the highest resistance was against Erythromycin.

\section{Discussion}

VAP is one of the prevalent infections with high mortality in patients hospitalized in ICUs of hospitals in most parts of the world. In this study, the most MDR isolated pathogens were Acinetobacter Baumannii and Pseudomonas aeruginosa. And the highest antibiotic sensitivity was observed in gram negative 
cases against ciprofloxacin, meropenem and imipenem, and in gram-positives against linezolid and ciprofloxacin. The mortality rate in this study was $38 \%$.

The prevalence of VAP in different regions is different according to several studies conducted $[4,8,9]$. However, the incidence of VAP in Asian countries is similar to the European countries. In the present study, it became clear that the incidence of VAP is lower in educational hospitals of Shiraz (especially Namazi). The reasons for the low incidence of pneumonia in Namazi Hospital in the intensive care units in comparison with the Asian countries, are as follows: 1- In the ICU of Namazi Hospital, the ratio of the number of nurses to the number of patients is 1:1, while in a study in India [10], the ratio has been 3:17. 2Nemazi Hospital has an active infection control committee and highly emphasizes the health of the personnel, especially washing the hands. 3- Care and protection of patients is done very carefully and patients are suctioned in a timely manner. 4. Traveling in ICU is prohibited, and patients do not associate with anyone else except the nurse and doctor. In general, the more standard become the interventions performed on hospitalized patients, and the more modern are the designs of the physical conditions of the hospitals to prevent VAP in a more modern form, the lower will be the frequency of having VAP.

Determining the factors that affect mortality extent will provide a better prognosis [11]. In our study, $45 \%$ of the patients with VAP received antibiotics before the incidence of pneumonia, of which $31.6 \%$ died. While of the remaining $54.8 \%$, only $8.7 \%$ died. In other words, patients who received antibiotics before the incidence of pneumonia had higher mortality. On the other hand, the most common microorganisms isolated from patients were Acinetobacter (28.8\%) and Pseudomonas (18.6\%) that were considered as part of MDR pathogens. Probably, the antimicrobial treatment prior to the onset of VAP has led to the selection of these MDR pathogens [12]. The most common pathogens in our study were Acinetobacter Baumannii (28.8\%) and then Pseudomonas aeruginosa (18.6\%). In general, the incidence of Acinetobacter in Asian countries is higher than other European countries. While the incidence of MRSA and $P$. aeruginosa in Asian countries is lower than the values reported in European countries [13]. The reason for the high incidence of Acinetobacter infection in Asian countries is not clear, but it may be due to temperature and humidity differences, because the warmer the environment and the higher the moisture, the more ideal become the conditions for the growth of Acinetobacter [14].

In this study, the mortality rate for the 20 individuals who received inappropriate experimental treatment was $23.4 \%$ and for the 27 subjects who received appropriate experimental treatment, it was $14.9 \%$. $P$. value was less than 0.05 . Therefore, there is a significant relationship between the quality of treatment and mortality. Several clinical studies have shown that experimental treatment of VAP with an appropriate antimicrobial regimen is associated with lower mortality [15-17]. In other words, the failure of antibiotic treatment is due to the presence of MDR pathogens [18]. In the prospective study by Fabrice Michel et al. [6] and also in the retrospective study of David L. Green [19], the patients whose experimental antibiotic therapy has been inappropriate, the changes in antibiotics after providing the culture response have not diminished the extent of mortality. In this study, patients who had received inappropriate experimental treatment had a higher mortality, however, this issue should also be taken into consideration that these patients had a critical condition of disease at the admitting time. Therefore, it cannot be 
properly demonstrated that inappropriate experimental treatment of hospital pneumonia has increased their mortality or is associated with their critical condition.

This finding show, overall $70.6 \%$ of the isolated Acinetobacters were sensitive to Meropenem and $76.5 \%$ of them were sensitive to amikacin, and in other words, they are the best coating for Acinetobacters. In the case of Pseudomonas, the highest susceptibilities were observed for meropenem, imipenem and ciprofloxacin $(90,80,70 \%)$ and appear to be the best treatment against this bacterium. Meropenem seems to be the best coating for both bacteria. Also in our study, the sensitivity of gram-positive bacteria against linezolid was (100\%). However, it is better to use vancomycin for treatment because linezolid is an alternative drug for treating VAP resulting from MRSA. Therefore, it is recommended that in the experimental treatment for MRSA treatment, vancomycin be used, which is considered the standard treatment for MRSA, and linezolid be stored for specific cases. Our data showed that most isolates (57\%) were related to MDR pathogens. The most common organisms in the last study in Shiraz, were Acinetobacter Baumannii, MRSA, and in the third place, the Pseudomonas aeruginosa, but in our study, Pseudomonas aeruginosa was ranked second.

\section{Limitations}

In order to calculate the prevalence of VAP, accurate statistics of hospitalized patients who received mechanical ventilation was necessary, which was not provided to us due to the hospital's security and ethical reasons.

\section{Abbreviations}

VAP, ventilator associated pneumonia; MDR, Multi Drug Resistant; ETTA, sampling techniques are endotracheal tube aspiration; BAZ, Bronchoalveolar lavage; PSB, Protected Specimen Brushing.

\section{Declarations}

\section{Consent to publish}

Not applicable.

\section{Availability of data and materials}

All relevant data are included in the manuscript.

\section{Funding}

This research was supported by the budget of research projects of the Shiraz University of medical sciences (approval code: 95-01-01-13716). Funding body were used to purchase equipment and tools.

\section{Acknowledgements}


Not applicable.

\section{Ethics approval and consent to participate}

This study was approved by the Ethics Committee of Shiraz University of Medical Sciences. The informed consent was obtained from all the participants, and informed consent obtained was written.

\section{Competing interests}

None of the authors have any competing interests.

\section{Authors' Contributions}

TKH, MSSA, NS, NH: design of study. NS, MAAD, MM, NH: acquisition of data. TKH, MSSA, NH: evaluation of data, preparation of the manuscript. NS, MAAD, MM, NH: assessment of data. All authors have read and approved the manuscript.

\section{References}

1. Foglia E, Meier M D, Elward A. 2. Clinical Microbiology Reviews. 2007;20(3):409-25.

2. Piazza 0 , Wang X. A translational approach to ventilator associated pneumonia. Clinical and translational medicine. 2014;3(1):26.

3. Bennett J E, Dolin R, Blaser M J. Principles and practice of infectious diseases: Elsevier Health Sciences; 2014.

4. Society A T, America I D S o. Guidelines for the management of adults with hospital-acquired, ventilator-associated, and healthcare-associated pneumonia. American journal of respiratory and critical care medicine. 2005;171(4):388.

5. Flanders S A, Collard H R, Saint S. Nosocomial pneumonia: state of the science. American journal of infection control. 2006;34(2):84-93.

6. Michel F, Franceschini B, Berger P, Arnal J-M, Gainnier M, Sainty J-M, et al. Early antibiotic treatment for BAL-confirmed ventilator-associated pneumonia. Chest. 2005;127(2):589-97.

7. Turton J F, Woodford N, Glover J, Yarde S, Kaufmann M E, Pitt T L. Identification of Acinetobacter baumannii by detection of the blaOXA-51-like carbapenemase gene intrinsic to this species. Journal of clinical microbiology. 2006;44(8):2974-6.

8. Chung D R, Song J-H, Kim S H, Thamlikitkul V, Huang S-G, Wang H, et al. High prevalence of multidrug-resistant nonfermenters in hospital-acquired pneumonia in Asia. American journal of respiratory and critical care medicine. 2011;184(12):1409-17.

9. Klompas M, Kleinman K, Khan Y, Evans R S, Lloyd J F, Stevenson K, et al. Rapid and reproducible surveillance for ventilator-associated pneumonia. Clinical infectious diseases. 2012;54(3):370-7.

10. Stebbings A E, Ti T Y, Tan W C. Hospital acquired pneumonia in the medical intensive care unit-a prospective study. Singapore Med J. 1999;40(8):508-12. 
11. Rello J, Ausino V, Ricart M, Castella J, Prats G. Impact of previous antimicrobial therapy on the etiology and outcome of ventilator-associated pneumonia. Chest. 1993;104(4):1230-5.

12. Fagon J-y, Chastre J, Domart Y, Trouillet J-L, Pierre J, Darne C, et al. Nosocomial pneumonia in patients receiving continuous mechanical ventilation: prospective analysis of 52 episodes with use of a protected specimen brush and quantitative culture techniques. American Review of Respiratory Disease. 1989;139(4):877-84.

13. Chawla R. Epidemiology, etiology, and diagnosis of hospital-acquired pneumonia and ventilatorassociated pneumonia in Asian countries. American journal of infection control. 2008;36(4):S93S100.

14. Jones R N. Microbial etiologies of hospital-acquired bacterial pneumonia and ventilator-associated bacterial pneumonia. Clinical Infectious Diseases. 2010;51(Supplement_1):S81-S7.

15. Luna C M, Videla A, Mattera J, Vay C, Famiglietti A, Vujacich P, et al. Blood cultures have limited value in predicting severity of illness and as a diagnostic tool in ventilator-associated pneumonia. Chest. 1999;116(4):1075-84.

16. Kollef M H, Sherman G, Ward S, Fraser V J. Inadequate antimicrobial treatment of infections: a risk factor for hospital mortality among critically ill patients. Chest. 1999;115(2):462-74.

17. Luna C M, Blanzaco D, Niederman M S, Matarucco W, Baredes N C, Desmery P, et al. Resolution of ventilator-associated pneumonia: prospective evaluation of the clinical pulmonary infection score as an early clinical predictor of outcome. Critical care medicine. 2003;31(3):676-82.

18. Luna C, Aruj P, Niederman M, Garzon J, Violi D, Prignoni A, et al. Appropriateness and delay to initiate therapy in ventilator-associated pneumonia. European Respiratory Journal. 2006;27(1):158-64.

19. Green $D$ L. Selection of an empiric antibiotic regimen for hospital-acquired pneumonia using a unit and culture-type specific antibiogram. Journal of intensive care medicine. 2005;20(5):296-301.

\section{Tables}

Table 1 Antibiotics were used to Test Antibiogram by Separating Different Isolated Bacteria 


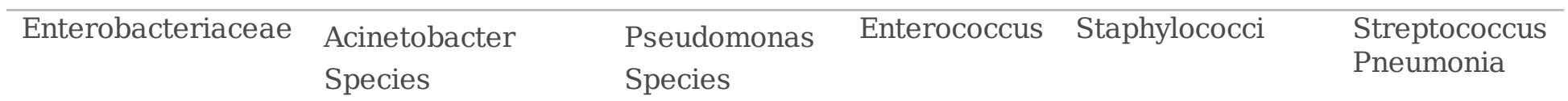

\begin{tabular}{|c|c|c|c|c|c|}
\hline Amikacin & Piperacillin & Piperacillin & Penicillin & Cefoxtin & Cefepime \\
\hline \multirow[t]{2}{*}{ Ciprofloxacin } & Ampicillin- & Piperacillin- & Vancomycin & Gentamycin & Clindamycin \\
\hline & Sulbactam & Tazobactam & & & \\
\hline Piperacillin & Ceftazidime & Ceftazidime & Erythromycin & Erythromycin & Erythromycin \\
\hline Piperacillin & Imipenem & Cefepime & Tetracycline & Tetracycline & Azitromycin \\
\hline \multicolumn{6}{|l|}{ Tazobactam } \\
\hline Imipenem & Meropenem & Azteronam & Ciprofloxacin & Ciprofloxacin & Tetracycline \\
\hline \multirow[t]{2}{*}{ Meropenem } & Amikacin & Imipenem & Linezolid & Trimethoprim & Levofloxacin \\
\hline & & & & Sulfamethoxazole & \\
\hline Fluoxetine & Minocycline & Meropenem & Teicoplanin & Linezolid & \\
\hline Ceftazidime & Ciprofloxacin & Amikacin & - & - & - \\
\hline \multirow[t]{2}{*}{ Cefipime } & Trimethoprim- & Ciprofloxacin & - & - & - \\
\hline & Sulfamethoxazole & & & & \\
\hline Ceftriaxone & - & - & - & - & - \\
\hline
\end{tabular}

Table 2 Primary antibiotic treatment and related mortality

\begin{tabular}{cccc}
\hline Variable & all & Death (\%) & P.value \\
\hline Empirical antibiotic treatment N (\%) & & & - \\
-Single antibiotic & 8 & $4(50 \%)$ & \\
-Combined antibiotics & 39 & $14(35 \%)$ & \\
Appropriateness of antibiotic treatment n (\%) & & & \\
- Appropriate & 27 & $7(25 \%)$ & 0.043 \\
- Inappropriate & 20 & $11(55 \%)$ & \\
Group of inititial antibiotic treatment & $\mathrm{n} \mathrm{( \% )}$ & & \\
-Early onset VAP & 18 & $5(27 \%)$ & - \\
-Late onset VAP & 29 & $13(44 \%)$ & \\
\hline
\end{tabular}

\section{Figures}




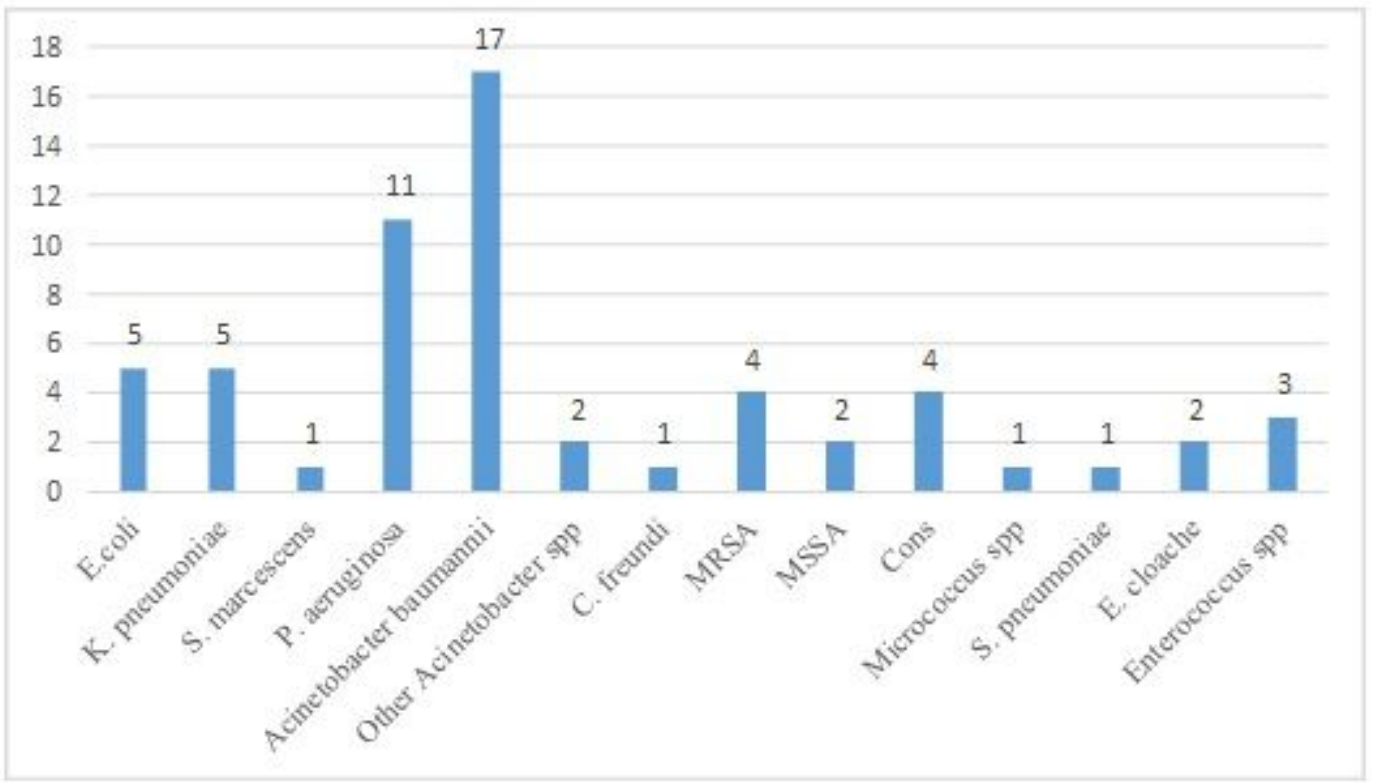

\section{Figure 1}

Frequency of detected bacteria of VAP patients admitted in internal wards and internal ICUs of educational hospitals of Shiraz 